Journal for ImmunoTherapy of Cancer

\title{
Molecular and immunological features of a prolonged exceptional responder with malignant pleural mesothelioma treated initially and rechallenged with pembrolizumab
}

\author{
Anna Minchom, ${ }^{1}$ Wei Yuan, ${ }^{1}$ Mateus Crespo, ${ }^{1}$ Bora Gurel, ${ }^{1}$ Ines Figueiredo, ${ }^{1}$ \\ Andrew Wotherspoon, ${ }^{2}$ Susana Miranda, ${ }^{1}$ Ruth Riisnaes, ${ }^{1}$ Ana Ferreira, ${ }^{1}$ \\ Claudia Bertan, ${ }^{1}$ Rita Pereira, ${ }^{1}$ Matt Clarke, ${ }^{1}$ Chloe Baker, ${ }^{1}$ Joo Ern Ang, ${ }^{1}$ \\ Nicos Fotiadis, ${ }^{3}$ Nina Tunariu, ${ }^{1}$ Suzanne Carreira (D) , ${ }^{1}$ Sanjay Popat, ${ }^{4}$ \\ Mary O'Brien, ${ }^{5}$ Udai Banerji, ${ }^{1}$ Johann de Bono, ${ }^{1}$ Juanita Lopez ${ }^{1}$
}

To cite: Minchom A, Yuan W, Crespo M, et al. Molecular and immunological features of a prolonged exceptional responder with malignant pleural mesothelioma treated initially and rechallenged with pembrolizumab. Journal for ImmunoTherapy of Cancer 2020;8:e00713. doi:10.1136/ jitc-2020-000713

- Additional material is published online only. To view please visit the journal online (http://dx.doi.org/10.1136/jitc2020-000713).

Accepted 05 February 2020

Check for updates

(c) Author(s) (or their employer(s)) 2020. Re-use permitted under CC BY-NC. No commercial re-use. See rights and permissions. Published by BMJ.

For numbered affiliations see end of article.

Correspondence to

Dr Anna Minchom;

anna.minchom@icr.ac.uk

\section{ABSTRACT}

Background This case represents an exceptional response to pembrolizumab in a patient with epithelioid mesothelioma with a further response on rechallenge. Case presentation A 77-year-old woman with advanced epithelioid mesothelioma extensively pretreated with chemotherapy demonstrated a prolonged response of 45 months to 52 cycles of pembrolizumab. On rechallenge with pembrolizumab, further disease stability was achieved. Serial biopsies and analysis by immunohistochemistry and immunofluorescence demonstrated marked immune infiltration and documented the emergency of markers of immune exhaustion. Whole exome sequencing demonstrated a reduction in tumor mutational burden consistent with subclone elimination by immune checkpoint inhibitor (CPI) therapy. The relapse biopsy had missense mutation in BTN2A1.

Conclusion This case supports rechallenge of programme death receptor 1 inhibitor in cases of previous CPI sensitivity and gives molecular insights.

\section{BACKGROUND}

Mesothelioma is a rare cancer of the pleura and mesothelial membranes associated with asbestos exposure and a poor prognosis. Subtypes include epithelioid, biphasic and sarcomatoid. A multimodal approach that may include surgery, radiotherapy and chemotherapy is often attempted for potentially resectable disease, but a proven survival benefit has not, as yet, been demonstrated. ${ }^{1}$ The majority of patients have inoperable disease. Treatment for inoperable disease has previously been with chemotherapy, though with relatively poor rates and duration of response, novel therapeutic strategies are required. ${ }^{2}$ Recent trials have assessed the utility of checkpoint inhibitor (CPI). The documentation of responses suggest that mesothelioma is a relatively 'immunogenic' tumor. ${ }^{34}$ Pembrolizumab is an antiprogramme death receptor 1 (PD-1) antibody investigated in mesothelioma. KEYNOTE-028 recruited 25 patients with PD-L1 (programmed deathligand 1) positive pleural mesothelioma and has reported interim results: objective response rate of $20 \%$, disease control rate of $52 \%$ and a median duration of response of 12.0 months (95\% CI of 3.7 to not reached).$^{5}$

\section{CASE PRESENTATION \\ Clinical background}

The patient is a 77-year-old Caucasian woman. She was diagnosed with a left epithelioid mesothelioma on video-assisted thorascopic biopsy in 2009 with pleurally based nodules in the left hemothorax on radiologic assessment. She underwent talc pleurodesis and four cycles of cisplatin and pemetrexed. Sixteen months later, she developed progressive disease and was treated on a trial of NGR-hTNF (a selective vascular inhibitor) for 4 months to disease progression. She underwent rechallenge with four cycles of pemetrexed and cisplatin, achieving disease stability for 11 months. She then received six cycles of carboplatin and gemcitabine achieving disease stability for 6 months.

From June 2014 to June 2016, she received 52 cycles of pembrolizumab (MK-3475) at a dose of $10 \mathrm{mg} / \mathrm{kg}$ every 2 weeks on a phase Ib clinic trial (KEYNOTE-028). The tumor biopsy fulfilled criteria for PD-L1 positivity as per trial protocol. She tolerated drug well 
A

B
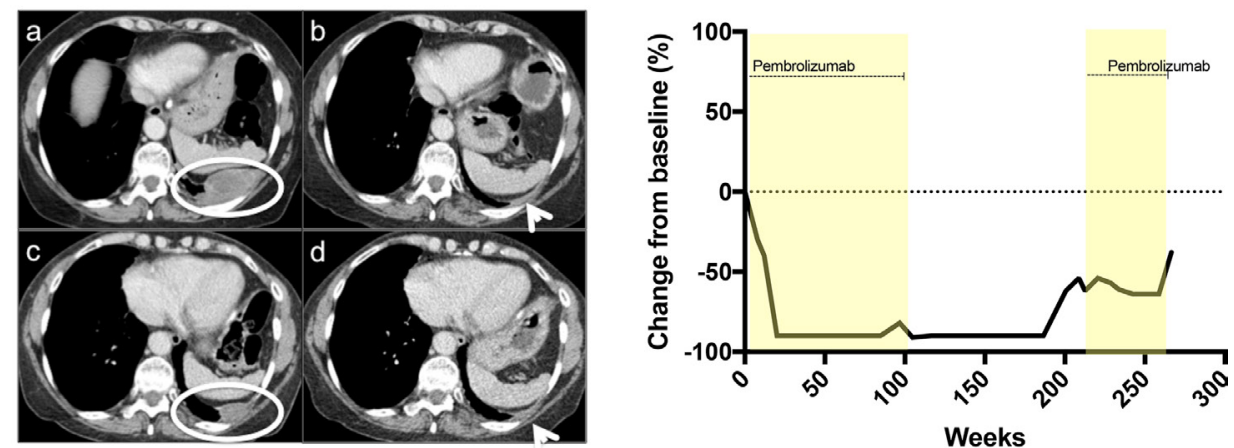

Figure 1 (A) Axial enhanced CT of thorax. Upper left panel a: baseline prior to commencing pembrolizumab trial (June 2014) with left posterior parietal malignant pleural disease (white circle). Upper right panel b: maintained partial response after 52 cycles pembrolizumab (April 2016) with minimal residual pleural thickening (white arrow). Lower left panel c: disease progression (July 2018) at site of previous disease along the left posterior parietal pleura (white circle). Lower right panel d: partial response in left parietal posterior pleural disease following three cycles pembrolizumab rechallenge. (B) Tumor response.

with immune-related adverse events of grade 2 pruritic rash and grade 1 mucositis, remaining ECOG (Eastern Cooperative Oncology Group) performance status 1. A partial response was seen on imaging after 3 months, with a $91 \%$ reduction in target lesions, which was maintained until June 2016 (figure 1). In April 2018, 21 months after completing 2years of pembrolizumab, she developed asymptomatic, small volume, radiologic disease progression and recommenced pembrolizumab on study, per

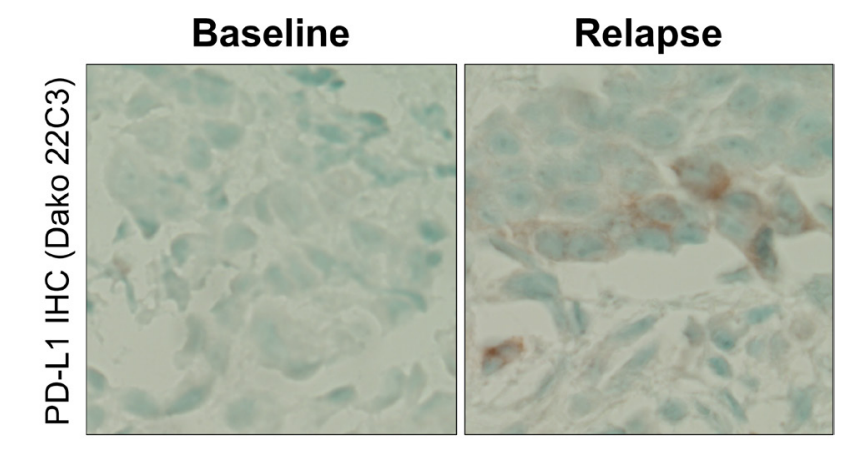

B

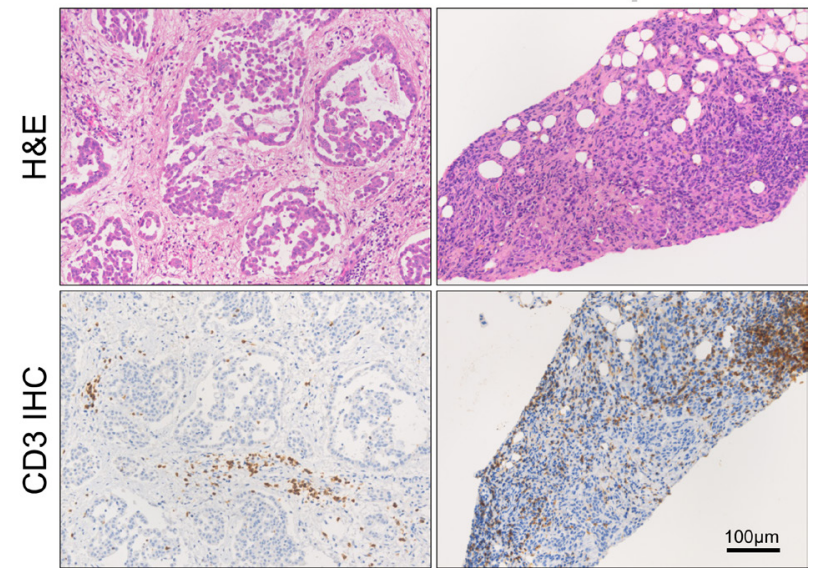

Figure 2 (A) PD-L1 IHC by Dako 22C3 in baseline (left panel) and relapse (right panel) biopsy. (B) CD3 by immunohistochemistry in baseline (left panel) and relapse (right panel) biopsy. protocol, on the same schedule. Following three cycles, a $12 \%$ reduction in tumor size by RECIST (Response evaluation criteria in solid tumour) criteria from the prerechallenge baseline was seen . Stable disease was maintained for 25 cycles when radiologic disease progression was confirmed.

\section{Laboratory correlates of immune response}

A left pleural biopsy from 2014, taken as baseline biopsy for KEYNOTE-028, and a left pleural biopsy taken in 2018 at relapse prior to pembrolizumab rechallenge were analysed. Histopathology was consistent with malignant epithelioid mesothelioma with cells expressing WT1, calretinin and HBME-1 and negative for BerEP4.

Immunohistochemistry for PD-L1 was performed using Dako 22C3 and Ventana SP263 clones (supplementary data for methods). PD-L1 staining was increased in the relapse compared with baseline biopsy $(1 \%-49 \%$ in relapse biopsy by SP263; figure 2).

CD3 immunohistochemistry was performed on baseline and relapse biopsies and intensity of staining quantified using the HALO software (supplementary data for methods). Intratumoral $\mathrm{T}$ cells were of a higher density in the relapse compared with baseline biopsy (2092.06/ $\mathrm{mm}^{2}$ vs $348.53 / \mathrm{mm}^{2}$ ) (figure 2 ).

A T cell panel immunofluorescence panel for CD4, CD4+ FOXP3+, CD8 and PanCK (pancytokeratin) was performed and analyzed with inForm Cell Analysis software (supplementary data for methods). Intratumoral CD8 $\mathrm{T}$ cells demonstrated an almost fivefold increase in relapse compared with baseline biopsy and CD4+ FOXP3+ Tcells demonstrated over a 30-fold increase in relapse compared with baseline (table 1 and figure 3 ).

\section{Genomics}

Whole exome sequencing (WES) was performed on both biopsy samples and a matched germline sample (supplementary data for methods). Tumor content was $80 \%$. The baseline biopsy had 0.92 somatic mutations per Mbp. The relapse biopsy had 0.26 . No mutations were found in key 
Table 1 Intratumoral T cell density (per $\mathrm{mm}^{2}$ ) on baseline and relapse biopsy by immunofluorescence

\begin{tabular}{lcc}
\hline T cell subset & $\begin{array}{l}\text { Baseline biopsy } \\
(\mathbf{2 0 1 4 )}\end{array}$ & $\begin{array}{l}\text { Relapse biopsy } \\
(\mathbf{2 0 1 8 )}\end{array}$ \\
\hline CD4 & 172.03 & 113.13 \\
CD4+ FOXP3+ & 2.62 & 88.89 \\
CD8 & 128.60 & 565.67 \\
All T cells & 303.26 & 767.69 \\
\hline
\end{tabular}

drivers such as BAP1, NF2, TP53, LATS2 and SETD2. On copy number variant analysis copy number alterations (CNAs) were apparent mostly similar in frequently altered genomic region between baseline and relapse biopsy such as chr8q gain, and chr3p and chr9p loss, but also some regions were different such as loss of heterozygosity on chr6q and chr4q in baseline only (figure 4). Three independent measurements of genomic instability (basis of loss of heterozygosity, telomeric allelic imbalance and large-scale state transitions) show baseline biopsy had unstable genome with higher HRD (homologous recombination deficiency) score. Immune-related somatic mutations are detailed in table 2 . All immunerelated somatic mutations present in the baseline biopsy were not present in the relapse biopsy. The relapse biopsy had missense mutation in BTN2A1 (c.1352G $>$ C).

\section{DISCUSSION AND CONCLUSION}

Dynamic immune changes and changes in tumor mutational burden (TMB) map the clinical response to pembrolizumab

The differences seen in levels of $\mathrm{T}$ cell infiltration between the two biopsies in this patient demonstrate the dynamic changes that occur in the context of CPI treated malignancy. Three cancer-immunity phenotypes have been described. The first is 'immune desert', which can be a result of tolerance, immunological ignorance or lack of priming. In this situation, no immune response is mounted to the cancer and little $\mathrm{T}$ cell infiltration is seen. The second is the 'immune excluded' tumor in which there is a barrier to immune cell migration to tumor caused by stromal interactions, vascular barrier and, again, no T cell infiltration is demonstrated. Third, the 'inflamed' tumor demonstrate infiltration by immune cells. Inhibitory factors (eg, PD-L1) and T cell exhaustion may still impair anticancer immunity in this setting. ${ }^{6} \mathrm{~T}$ cell exhaustion describes a progressive loss of $\mathrm{T}$ cell function occurring on persistent antigen presentation. ${ }^{7}$ Relapse biopsies in this patient demonstrate increased immune cell infiltrate of CD3 CD8 and CD4 T cells, compared with baseline. This is indicative of immune activation as a result of the primary immunotherapy treatment (a move from an immune desert to inflamed tumor) and is consistent with the prolonged response. However, there is also an increase in FOXP3 positive T cell, a marker of regulatory T cells and PD-L1. Therapeutic targeting of PD-1 is known to effect regulatory $\mathrm{T}$ cell function but not overall number. ${ }^{8}$ We may consider the increase in regulatory $\mathrm{T}$ cells a marker of immune exhaustion. These markers of immune exhaustion represent emerging resistance to immunotherapy as evidenced by the clinical progression. Despite these markers of immune exhaustion, a response to pembrolizumab rechallenge was achieved, thus resistance to immunotherapy was not complete. The finely tuned balance of immunostimulatory and immunosuppressive elements demonstrated in these sequential biopsies in combination with the radiologic data present a compelling visualization of immune activation and exhaustion and clinical implications. A disadvantage of this study is that single biopsies were taken, and there may be heterogeneity of immune infiltrates throughout the tumor burden. Ongoing trials address the potential in mesothelioma for drug combinations to move tumors to the inflamed phenotype and overcome CPI resistance. Preclinal

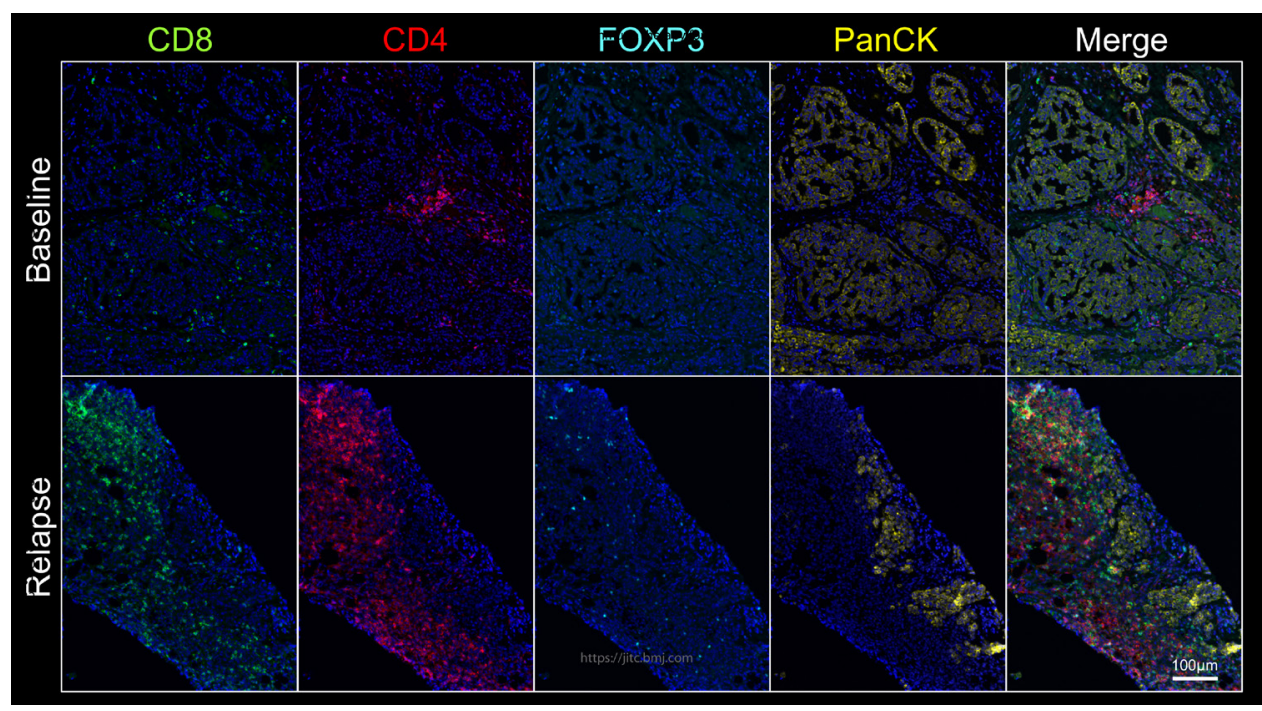

Figure 3 Multicoloured immunofluorescence panel for T cells in baseline (upper panels) and relapse (lower panels) biopsy. 

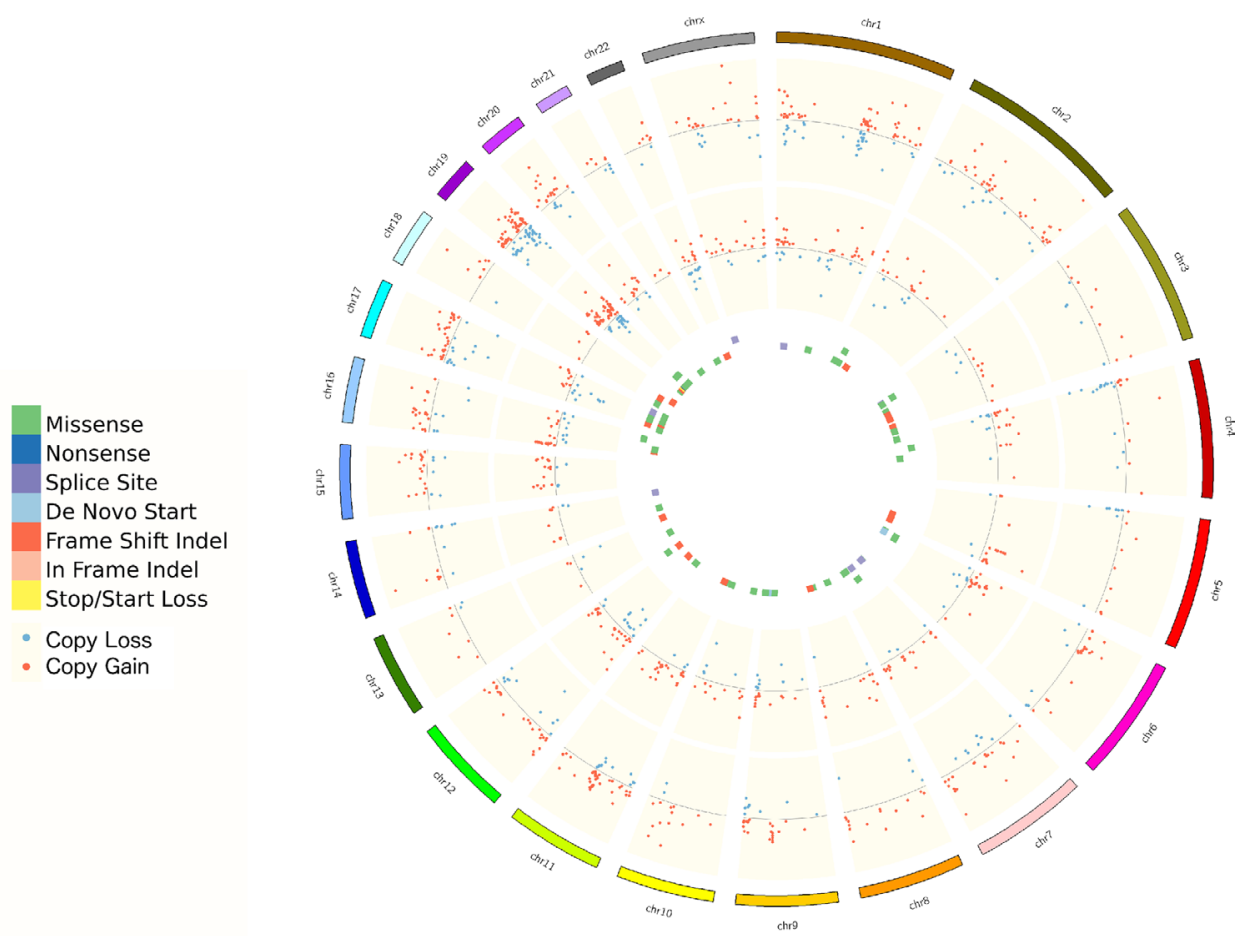

Figure 4 Circos plot of CNA and somatic mutations. From outermost to innermost track: progression sample CNA (log2R), baseline sample CNA (log2R), progression sample mutations and baseline sample mutations. CNAs, copy number alterations.

evidence suggests chemotherapy causes a degree of immune activation, ${ }^{9}$ and studies propose rational combination and sequencing of chemotherapy and CPI to achieve this end. The phase II DREAM study of durvalumab in combination with pemetrexed and cisplatin gave an objective treatment response rate of $48 \%$, and a phase III is planned. ${ }^{10}$

The reduction in the number of somatic mutations between two samples can suggest subclones eliminated by pembrolizumab. This phenomenon is well described previously in patients with melanoma treated with nivolumab. ${ }^{11}$ On treatment with CPI, immunoediting occurs where tumor cells expressing neoantigens targeted by activated $\mathrm{T}$ cells are lost. ${ }^{12}$ The resulting loss of cancer heterogeneity results in more homogenous cancer cell population and a lower rate of somatic mutations and a lower TMB.

\section{The case in context as a long-term responder to pembrolizumab and chemotherapy}

What is remarkable about this patient's initial response is the depth and duration. The relapse of disease occurred 21 months after the last dose of pembrolizumab. A recent paper suggests nivolumab can be detected more

Table 2 Immune-related somatic mutations on baseline and relapse biopsy

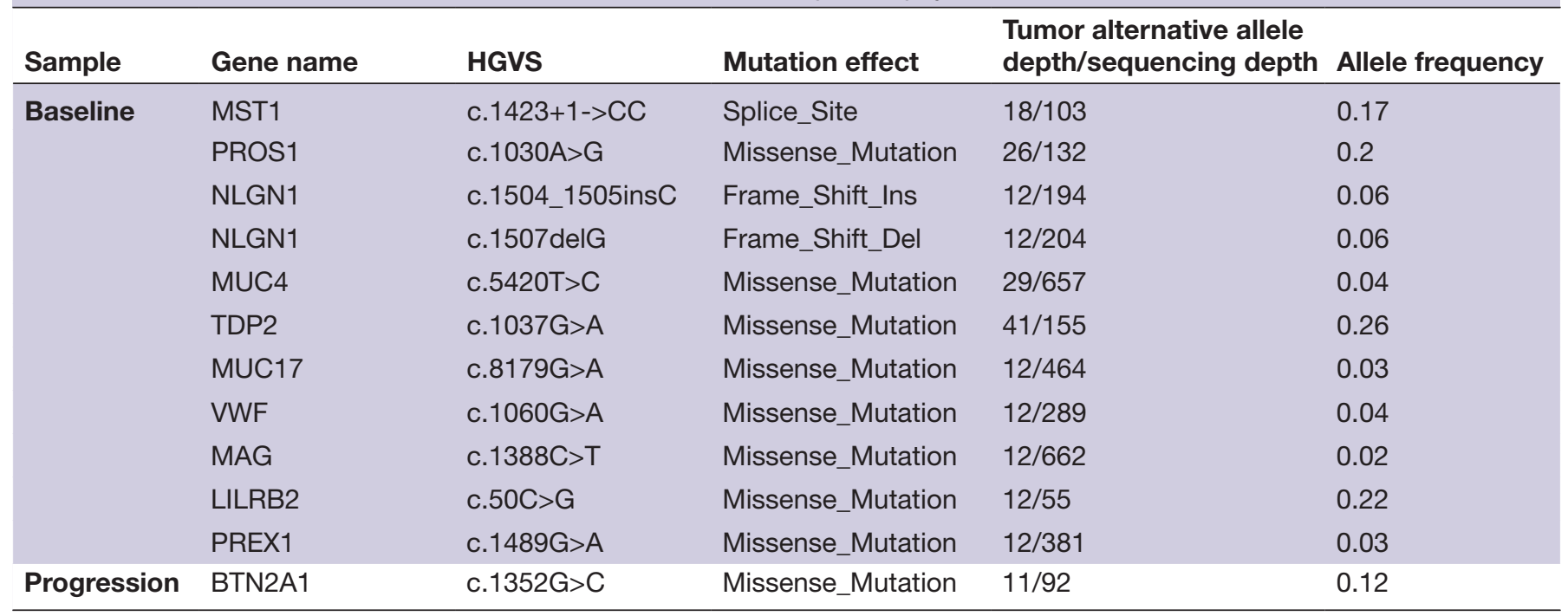


than 20 weeks following administration, which is longer than might be anticipated from previous pharmacokinetic data. ${ }^{13}$ Nevertheless, the relapse in this patient occurred long after the elimination of all residual drug. Most CPI trials demonstrate, a 'tail to the curve' with a small number of patients who achieve a prolonged response. ${ }^{14}$ Study of these 'exceptional responders' can potentially inform on biologic features that mark prolonged response and be hypothesis generating for further research into mechanisms of drug resistance and sensitivity.

WES results reveal a TMB low tumor. Mesothelioma is classically a TMB low tumor. Analysis of 74 cases revealed a somatic mutation rate of less than two per megabase in all but one case. ${ }^{15}$ Moreover, in keeping with published data is the CNA seen in this case. Others report frequent CNA in keeping with mesothelioma being driven by loss of tumor suppressors rather than an oncogenic driven cancer. ${ }^{15}$ Transcriptome analysis was not performed. Others have identified expression of the negative CPI VISTA commonly in mesothelioma, which may have implications on CPI response. ${ }^{15}$

Proposed resistance mechanisms to CPI are numerous and may be multifactorial. ${ }^{16}$ The only immune-related mutated gene evidenced in the relapse biopsy was BTN2A1. This is a T cell immunomodulatory molecule coregulated with MHC class II. ${ }^{17}$ Its role in CPI resistance is not described. As the BTN2A mutation was seen on the relapse biopsy (postrelapse but prerechallenge), the implications of the mutation (if any) is unclear, whether having a role in emerging resistance or sensitivity to rechallenge.

It is also interesting to consider the patient's prior response to chemotherapy. She achieved an unusual (though not unique) 16-month progression free survival with first-line cisplatin pemetrexed chemotherapy and further response on two chemotherapy rechallenges. The phase II MAPS2 trial of nivolumab or nivolumabipilimumab in relapsed mesothelioma included an post hoc analysis showing that in the nivolumab group patients who had relapse at least 3 months after pemetrexed chemotherapy had a small survival benefit. ${ }^{18}$ Whether these findings are replicated in other trials and whether this simply represents a more globally indolent disease or whether there is a biologic rationale for chemotherapy response correlating with benefit from CPI remains to be seen.

\section{The case in context as a response to pembrolizumab rechallenge}

This patient's cancer is also exceptional in its responsiveness to pembrolizumab on rechallenge. This phenomena has not be studied in detail. Though others report the potential for a response with CPI rechallenge, ${ }^{19}$ this is the first report, to our knowledge, of disease response on CPI rechallenge in mesothelioma.

\section{CONCLUSION}

In conclusion, this case represents a prolonged response to pembrolizumab in a patient with epithelioid mesothelioma to PD-1 inhibition with further durable clinical benefit on rechallenge. This supports trial data from KEYNOTE-028 and others that mesothelioma can be responsive to CPI. In this case, no reason for prolonged immune sensitivity was identified. The tumor, though PD-L1 positive, did not demonstrate a very high level of PD-L1 expression. WES did not shed light on reasons for prolonged sensitivity to CPI, chromothripsis and loss of heterozygosity are not fully assessed on WES and epigenetic modifications such as methylation are not evaluated by WES.

Serial biopsies demonstrate both the primary immune activation and emerging immune exhaustion. Future research may shed light on the mechanisms of resistance and pave the way for drug combinations to overcome CPI resistance. Cases such as this support attempts to retreat with CPI if a patient clinical condition allows. Further research into the degree to which a 'partially exhausted' immune environment can be reactivated by further stimulation are warranted.

\section{Author affiliations}

${ }^{1}$ Drug Development Unit, Royal Marsden Hospital/ Institute of Cancer Research,

Sutton, UK

${ }^{2}$ Department of Histopathology, Royal Marsden Hospital, London, UK

${ }^{3}$ Department of Radiology, Royal Marsden Hospital, London, UK

${ }^{4}$ Lung Unit, Royal Marsden Hospital, London, UK

${ }^{5}$ Lung Unit, Royal Marsden Hospital, Sutton, UK

Contributors AM: data collection, data analysis, data interpretation and manuscript preparation. WY: data analysis and data interpretation. MC, BG, IF, AW, SM, RR, AF, $\mathrm{CB}, \mathrm{RP}, \mathrm{MC}$ and $\mathrm{CB}$ : laboratory analysis. JEA: data collection. NF: tissue specimens. $\mathrm{NT}$ : radiology data preparation and interpretation. SC: laboratory analysis and data interpretation. SP, M0, UB and JdB: data review and interpretation. JL: data analysis, data interpretation and manuscript preparation. All authors: manuscript review.

Funding This study represents independent research supported by the National Institute for Health Research (NIHR) Biomedical Research Centre at the Royal Marsden NHS Foundation Trust and the Institute of Cancer Research.

Disclaimer The views expressed are those of the authors and not necessarily those of the NIHR or the Department of Health and Social Care.

Competing interests AM: honoraria from FARON and Bayer. AW: advisory boards for Bayer, Bristol-Myers Sqibb and Celgene. SP: honoraria from Boehringer Ingelheim, AstraZeneca, Roche, Takeda and Chugai Pharma; advisory boards from Boehringer Ingelheim, AstraZeneca, Roche, Novartis, Pfizer, Bristol-Myer Squibb, MSD, Guardant Health, Abbvie, EMD Serono and Takeda; expenses from Boehringer Ingelheim, Bristol-Myer Squibb and Merck Sharp \& Dohme. M0: advisory boards for MSD, Abbvie, BMS, BI, Pierre Fabre. UB: honoraria from Astellas, Novartis, Karus Therapeutics, Phoenix Solutions, Eli Lilly, Astex and Vernalis; funding for phase I investigator-initiated trials from Onyx Pharmaceuticals, BTG International, Chugai, Astrazeneca and Verastem. JdB: personal fees and non-financial support from Astellas Pharma, Genentech/Roche, Pfizer, Sanofi, Bayer, Boehringer Ingelheim, Merck Serono and Merck Sharp \& Dohme; grants, personal fees and non-financial support from AstraZeneca; non-financial support from Genmab, GlaxoSmithKline, Orion Pharma GmbH, Qiagen, Taiho Pharmaceutical and Vertex. In addition, JdB has a patent Abiraterone Rewards to Inventors with royalties paid to institution, no personal income and a patent PARP inhibitors and DNA repair defects with royalties paid to institution, no personal income. JL: research funding from Roche Genentech, Genmab and Basilea Travel from Basilea.

Patient consent for publication Obtained. 
Ethics approval Ethical approval was obtained from local research and ethics committee at the Royal Marsden Hospital (CCR ref: 3171).

Provenance and peer review Not commissioned; externally peer reviewed.

Open access This is an open access article distributed in accordance with the Creative Commons Attribution Non Commercial (CC BY-NC 4.0) license, which permits others to distribute, remix, adapt, build upon this work non-commercially, and license their derivative works on different terms, provided the original work is properly cited, appropriate credit is given, any changes made indicated, and the use is non-commercial. See http://creativecommons.org/licenses/by-nc/4.0/.

\section{ORCID iD}

Suzanne Carreira http://orcid.org/0000-0002-5077-5379

\section{REFERENCES}

1 Baas P, Fennell D, Kerr KM, et al. Malignant pleural mesothelioma: ESMO clinical practice guidelines for diagnosis, treatment and follow-up. Annals of Oncology 2015;26:v31-9.

2 Scherpereel A, Wallyn F, Albelda SM, et al. Novel therapies for malignant pleural mesothelioma. Lancet Oncol 2018;19:e161-72.

3 Dozier J, Zheng H, Adusumilli PS. Immunotherapy for malignant pleural mesothelioma: current status and future directions. Transl. Lung Cancer Res. 2017;6:315-24.

4 Yap TA, Aerts JG, Popat S, et al. Novel insights into mesothelioma biology and implications for therapy. Nat Rev Cancer 2017:17:475-88.

5 Alley EW, Lopez J, Santoro A, et al. Clinical safety and activity of pembrolizumab in patients with malignant pleural mesothelioma (KEYNOTE-028): preliminary results from a non-randomised, openlabel, phase 1B trial. Lancet Oncol 2017;18:623-30.

6 Chen DS, Mellman I. Elements of cancer immunity and the cancerimmune set point. Nature 2017;541:321-30.

7 Yi JS, Cox MA, Zajac AJ. T-Cell exhaustion: characteristics, causes and conversion. Immunology 2010;129:474-81.
8 Toor SM, Syed Khaja AS, Alkurd I, et al. In-vitro effect of pembrolizumab on different T regulatory cell subsets. Clin Exp Immunol 2018;191:189-97.

9 Emens LA, Middleton G. The interplay of immunotherapy and chemotherapy: harnessing potential synergies. Cancer Immunol Res 2015;3:436-43.

10 Nowak A, Kok P, Lesterhuis W, et al OA08.02 DREAM - A Phase 2 Trial of Durvalumab with First Line Chemotherapy in Mesothelioma: Final Result. Journal of Thoracic Oncology 2018;13:S338-9.

11 Riaz N, Havel JJ, Makarov V, et al. Tumor and microenvironment evolution during immunotherapy with nivolumab. Cell 2017;171:934-49.

12 O'Donnell JS, Teng MWL, Smyth MJ. Cancer immunoediting and resistance to T cell-based immunotherapy. Nat Rev Clin Oncol 2019:16:151-67.

13 Osa A, Uenami T, Koyama S, et al. Clinical implications of monitoring nivolumab immunokinetics in non-small cell lung cancer patients. JCl Insight 2018;3.

14 Gettinger S, Horn L, Jackman D, et al. Five-Year follow-up of nivolumab in previously treated advanced Non-Small-Cell lung cancer: results from the CA209-003 study. JCO 2018;36:1675-84.

15 Hmeljak J, Sanchez-Vega F, Hoadley KA, et al. Integrative molecular characterization of malignant pleural mesothelioma. Cancer Discov 2018;8:1548-65.

16 Sharma P, Hu-Lieskovan S, Wargo JA, et al. Primary, adaptive, and acquired resistance to cancer immunotherapy. Cell 2017;168:707-23.

17 Sarter K, Leimgruber E, Gobet F, et al. Btn2a2, a T cell immunomodulatory molecule coregulated with MHC class II genes. $J$ Exp Med 2016;213:177-87.

18 Scherpereel A, Mazieres J, Greillier L, et al. Nivolumab or nivolumab plus ipilimumab in patients with relapsed malignant pleural mesothelioma (IFCT-1501 MAPS2): a multicentre, openlabel, randomised, non-comparative, phase 2 trial. Lancet Oncol 2019;20:239-53.

19 Bernard-Tessier A, Baldini C, Martin P, et al. Outcomes of long-term responders to anti-programmed death 1 and anti-programmed death ligand 1 when being rechallenged with the same anti-programmed death 1 and anti-programmed death ligand 1 at progression. Eur $J$ Cancer 2018;101:160-4. 